\title{
Associação entre qualificação profissional de fisioterapeutas e a percepção sobre o tratamento de osteoartrite de joelho: um estudo transversal
}

\author{
Association between professional qualification \\ of physical therapists and perception of knee \\ osteoarthritis treatment: a cross-sectional study
}

\author{
Nívea Renata Oliveira Monteiro' ${ }^{1}$ (1) \\ Débora Juliana do Rosário² \\ Vânia Tiê Koga Ferreira 3 (1) \\ Daniela Gonçalves Ohara ${ }^{4}$ (1)
}

\author{
Maycon Sousa Pegorari5 \\ Império Lombardi Júnior ${ }^{6}$ (1) \\ Areolino Pena Matos ${ }^{7}$ (1)
}

1-5Universidade Federal do Amapá (Macapá). Amapá, Brasil. ni.vea.ap@hotmail.com, deborajuliana720@gmail.com, vaniatie@gmail.com, dani_ohara@hotmail.com, mayconpegorari@yahoo.com.br

שUniversidade Federal de São Paulo (São Paulo). São Paulo, Brasil. imperiolombardi@gmail.com ${ }^{7}$ Autor para correspondência. Universidade Federal do Amapá (Macapá). Amapá, Brasil. areolino.matos@gmail.com

RESUMO I INTRODUÇÃO: A Osteoartrite (OA) é a forma mais prevalente de artrite e requer intervenção terapêutica adequada. A Fisioterapia desempenha um importante papel no manejo desta doença. No entanto, pouco se sabe sobre as crenças e intervenções de fisioterapeutas que tratam OA no Brasil e fatores relacionados às decisões clínicas. OBJETIVO: Determinar se existe associação entre a qualificação profissional de fisioterapeutas na região Norte do Brasil e a percepção de eficiência ou ineficiência de intervenções terapêuticas para OA de joelho. MATERIAIS E MÉTODOS: Uma pesquisa transversal online foi realizada com fisioterapeutas de cinco estados do Norte do Brasil e a percepção sobre 20 intervenções foi avaliada por meio de um caso hipotético de OA de joelho. O teste qui-quadrado e regressão logística binária com nível de significância de $5 \%$ foram utilizados para a análise estatística. RESULTADOS: 370 profissionais com média de idade de $32,16 \pm 6,89$ responderam a pesquisa. Houve associação significativa entre qualificação e percepção de eficiência das intervenções. Fisioterapeutas apenas graduados apresentaram menos chances de reconhecer ultrassom, ondas curtas, neuroestimulação elétrica transcutânea e estimulação elétrica, crioterapia, calor, fortalecimento muscular, kinesio taping e repouso como intervenções ineficazes. O mesmo ocorreu com as intervenções ultrassom, fortalecimento muscular e kinesio taping para especialistas. CONCLUSÃo: O menor grau acadêmico leva à uma chance menor de perceber como pouco eficientes ou ineficientes algumas intervenções não recomendadas para OA de joelho.

PALAVRAS-CHAVE: Inquéritos e questionários. Osteoartrite de joelho. Prática profissional. Modalidades de fisioterapia.
ABSTRACT | INTRODUCTION: Osteoarthritis (OA) is the most prevalent form of arthritis, which requires appropriate therapeutic intervention. Physical therapy plays a central role in its management. However, little is known about the beliefs and interventions of physical therapists who treat $O A$ in Brazil and factors related to clinical decisions. OBJECTIVE: To determine whether there is an association between the professional qualification of physical therapists in the North Region of Brazil and their perception of efficiency or inefficiency of therapeutic interventions for knee OA. METHODS: A cross-sectional online survey was conducted with physical therapists from five states in northern Brazil, and their perception about 20 interventions was assessed through a hypothetical case of knee OA. Statistical analyses were performed using the chi-square test and binary logistic regression with a significance level of 5\%. RESULTS: 370 professionals with a mean age of $32.16 \pm 6.89$ responded to the survey. There was a significant association between qualification and perceived efficiency of interventions. Graduated physiotherapists were less likely to recognize ultrasound, short waves, transcutaneous electrical neurostimulation and electrical stimulation, cryotherapy, heat, muscle strengthening, kinesio taping, and rest as ineffective interventions. The same occurred for ultrasound, muscle strengthening, and kinesiology tape for specialists. CONCLUSION: The lower academic degree leads to a lower chance to perceive as poorly efficient or inefficient some not recommended interventions for knee OA.

KEYWORDS: Health care surveys. Osteoarthritis, knee. Professional practice. Physical therapy modalities. 


\section{Introdução}

A Osteoartrite (OA) é a doença articular mais comum do mundo desenvolvido e a principal causa de incapacidade crônica de articulações periféricas ${ }^{1,2}$. Joelhos com OA sintomático são muito prevalentes entre idosos no mundo todo (10\% a $30 \%$ ) especialmente em regiões rurais cujas demandas físicas de trabalho são altas $^{3}$. Nesta faixa etária, a doença é a causa mais comum de incapacidade, incluindo dor e limitação nas Atividades de Vida Diária (AVDs) $)^{4,5}$.

Devido ao grande impacto na função individual e repercussão social, há reconhecida necessidade de tratamentos de alta qualidade. Por isso, várias diretrizes de prática clínica para este tipo de cuidado são publicadas regularmente ${ }^{6-8}$. Comumente estão disponíveis condutas cirúrgicas, farmacológicas e as não-farmacológicas, nas quais os tratamentos da Fisioterapia estão incluídos e são considerados as principais escolhas terapêuticas para a doença ${ }^{8}$.

Condutas e crenças do fisioterapeuta para o tratamento de OA do joelho já foram investigadas em inquéritos anteriores. Holden et al. notaram que pouco mais da metade dos profissionais (56\%) concordaram que exercícios melhoram as condições do joelho e consideravam sua prática uma reponsabilidade do paciente ${ }^{2}$. Em outra pesquisa, observou-se que apesar da maioria dos fisioterapeutas utilizarem exercícios e educação do paciente, recursos como a eletroterapia e ultrassom ainda são utilizados ${ }^{10}$. Foi demonstrado também que fatores relacionados ao profissional podem ter relação com essas decisões clínicas, a idade do profissional esteve associada com a sua escolha terapêutica para o tratamento de OA de joelho em dois estudos anteriores, onde fisioterapeutas mais velhos foram mais prováveis de utilizar intervenções não recomendadas para esta condição ${ }^{10,11}$.

Pouco se sabe sobre a prática de fisioterapeutas brasileiros no tratamento de OA de joelho e potenciais fatores modificáveis que podem estar associados à percepção de eficiência ou ineficiência de intervenções terapêuticas para o tratamento desta condição. Estudos com esta finalidade podem auxiliar na compreensão acerca do tipo e qualidade do atendimento que pacientes estão recebendo e ajudam a projetar o alcance das evidências científicas entre profissionais no país.
Portanto, o objetivo deste estudo foi determinar se existe associação entre qualificação profissional de fisioterapeutas na região Norte do Brasil e suas percepções de eficiência ou ineficiência de intervenções terapêuticas para OA de joelho.

Este é o primeiro estudo conduzido no Brasil com este objetivo e nossa hipótese é de que profissionais com níveis diferentes de formação interpretem de forma distinta a eficiência de intervenções terapêuticas.

\section{Materiais e métodos}

\section{Tipo de estudo e amostra}

Inquérito eletrônico descritivo, transversal e analítico, com abordagem quantitativa, descrito de acordo com a iniciativa Strengthening the Reporting of Observational Studies in Epidemiology (STROBE) e aprovado pelo Comitê de Ética em Pesquisa (Parecer: 1.989.417; CAAE 62673016.4.0000.0003).

O inquérito eletrônico foi conduzido com a participação de fisioterapeutas que tratam pacientes com OA de joelho, registrados no Conselho de Fisioterapia e atuantes em cinco estados da região Norte do Brasil.

O tamanho da amostra foi calculado com base em uma prevalência de $50 \%$. O cálculo utilizou como população total o número de contatos eletrônicos (6.285) com erro amostral de 5\%, nível de confiança de $95 \%$, chegando ao número mínimo de 363 respostas necessárias. A utilização de uma prevalência de 50\% para o cálculo amostral é uma estratégia conservadora, considerando que a prevalência de certos desfechos é desconhecida ou se pretende-se planejar um estudo com diferentes desfechos de interesse. Desta forma, para uma dada precisão, a prevalência de 50\% para um determinado desfecho fornece o maior tamanho amostral possível em uma estimativa.

\section{Instrumento de coleta de dados}

Os autores elaboraram um inquérito eletrônico com 13 questões administradas por meio do website SurveyMonkeyTM (www.surveymonkey.com). O instrumento, aplicado entre julho de 2017 e fevereiro de 2018 , foi previamente analisado por um grupo de seis 
fisioterapeutas docentes e clínicos, especialistas em fisioterapia musculoesquelética e/ou reumatológica, com experiência mínima de 10 anos no atendimento a pacientes com OA de joelho. O grupo avaliou o conteúdo, possíveis ambiguidades, termos técnicos, terminologias, tempo gasto para responder e a estrutura da ferramenta. As sugestões e modificações foram examinadas e incluídas na versão final. Um estudo piloto foi realizado entre 15 docentes para aprimorar e avaliar os procedimentos de envio e coleta de dados.

O questionário continha: um texto inicial, que demandava do profissional atendimento de no mínimo um paciente por mês com OA de joelho. Mais quatro seções com: 1) Dados sócio demográficos; 2 ) Informações acerca da formação profissional e atuação clínica; 3) Análise do profissional acerca da efetividade de intervenções em fisioterapia utilizadas no tratamento da OA de joelho (para estas perguntas utilizou-se um caso hipotético de uma paciente com dor e disfunção articular, 20 intervenções terapêuticas pré-definidas foram fornecidas como resposta); 4) Fontes de informação e uso da Prática Baseada em Evidências para escolha de tratamentos.

\section{Procedimentos}

Os dados foram coletados via endereço eletrônico. O convite à participação no estudo e o inquérito eletrônico foram enviados para todos os fisioterapeutas dos cinco Estados da Região Norte do país. Um prazo de resposta de até três semanas foi estabelecido.

\section{Análise estatística}

O banco de dados foi importado para o programa Statistical Package for Social Sciences (SPSS Versão 25.0. IBM Inc, Chicago, IL USA). As variáveis categóricas foram analisadas por meio de frequências absolutas e percentuais, para as numéricas, média e desvio-padrão. Para verificar a associação das variáveis desfecho de interesse (tipo de tratamento e opções: eficiente/muito eficiente ou ineficiente/pouco eficiente) com as variáveis independentes (graduação, especialização/residência e mestrado/doutorado), utilizou-se o teste qui-quadrado e para as estimativas das razões de chance, o modelo de regressão logística binária, considerando um nível de significância de $5 \%(p<0,05)$ e intervalo de confiança (IC) de 95\%.

\section{Resultados}

A tabela 1 apresenta as características dos fisioterapeutas que responderam ao estudo. 370 profissionais responderam o inquérito eletrônico, com taxa de resposta de 5,88\%. A maioria dos profissionais da região eram mulheres (64,59\%), com média de idade de 32,16 \pm 6,89 anos, com especialização lato sensu ou residência $(58,11 \%)$ e menos de $14 \%$ possuía pós-graduação em nível stricto sensu. A participação dos profissionais por estado foi de $42,9 \%$ provenientes do Pará, $23,2 \%$ de respondentes do Amazonas, $17,2 \%$ do Amapá, $11,8 \%$ responderam do Tocantins e 4,5,5 do estado de Roraima.

Tabela 1. Características dos profissionais que responderam ao inquérito

\begin{tabular}{|c|c|}
\hline Variáveis & Amostra total $(n=370)$ \\
\hline Idades (anos $\pm \mathrm{DP}$ ) & $32,16 \pm 6,89$ \\
\hline \multicolumn{2}{|l|}{ Gênero } \\
\hline Masculino & $131(35,4)$ \\
\hline Feminino & $239(64,5)$ \\
\hline Tempo de licença profissional (anos \pm DP) & $6,8 \pm 5,61$ \\
\hline \multicolumn{2}{|l|}{ Qualificação } \\
\hline Graduação & $101(27,3)$ \\
\hline Especialização/Residência & $215(58,1)$ \\
\hline Mestrado & $47(12,0)$ \\
\hline Doutorado & $7(1,9)$ \\
\hline \multicolumn{2}{|c|}{$\begin{array}{l}\text { Você possui treinamento específico para o tratamento de } O A \text { de } \\
\text { joelho? }\end{array}$} \\
\hline $\operatorname{sim}$ & $245(66,2)$ \\
\hline Não & $125(33,7)$ \\
\hline
\end{tabular}

DP: Desvio padrão; OA: Osteoartrite 
A Tabela 2 apresenta a associação entre a titulação profissional e a percepção da eficiência ou ineficiência das intervenções. Independente da titulação, o maior percentual de fisioterapeutas considerou eficiente ou muito eficiente as terapias de fortalecimento muscular, ultrassom, TENS e eletroestimulação e crioterapia. Para as terapias ondas curtas, kinesio taping e repouso, o grupo de graduados e especialistas considerou eficiente ou muito eficiente, enquanto que o grupo de mestres e doutores, ineficiente ou pouco eficiente. Fisioterapeutas graduados consideraram as terapias de calor superficial e turbilhão como eficientes ou muito eficientes e aqueles com especialização/residência e mestrado/doutorado ineficientes ou pouco eficientes $(p<0,05)$.

Tabela 2. Associação entre qualificação profissional e percepção de eficiência ou ineficiência das intervenções

\begin{tabular}{|c|c|c|c|c|c|c|c|c|c|}
\hline \multirow{4}{*}{$\begin{array}{l}\text { Variáveis } \\
\text { Terapia }\end{array}$} & \multicolumn{6}{|c|}{ Qualificação profissional } & & & \multirow{4}{*}{$P$} \\
\hline & \multicolumn{2}{|c|}{ Graduação } & \multicolumn{2}{|c|}{$\begin{array}{l}\text { Especialização/ } \\
\text { Residência }\end{array}$} & \multicolumn{2}{|c|}{$\begin{array}{l}\text { Mestrado/ } \\
\text { Doutorado }\end{array}$} & \multicolumn{2}{|c|}{ Total } & \\
\hline & $\mathrm{ME} / \mathrm{E}$ & I/PE & $\mathrm{ME} / \mathrm{E}$ & I/PE & $\mathrm{ME} / \mathrm{E}$ & I/PE & $\mathrm{ME} / \mathrm{E}$ & I/PE & \\
\hline & \multicolumn{2}{|c|}{$\mathrm{n}(\%)$} & \multicolumn{2}{|c|}{$\mathrm{n}(\%)$} & \multicolumn{2}{|c|}{$n(\%)$} & \multicolumn{2}{|c|}{$\mathrm{n}(\%)$} & \\
\hline Ultrassom & $93(30,9)$ & $8(11,6)$ & $172(57,1)$ & $43(62,3)$ & $36(12)$ & $18(26,1)$ & $301(81,4)$ & $69(18,6)$ & $0,001^{*}$ \\
\hline LASER & $81(28,2)$ & $20(24,1)$ & $168(58,5)$ & $47(56,6)$ & $38(13,2)$ & $16(19,3)$ & $287(77,6)$ & $83(22,4)$ & 0,359 \\
\hline Ondas curtas & $66(36)$ & $35(20,6)$ & $109(54,5)$ & $106(62,4)$ & $25(12,5)$ & $29(17,1)$ & $200(54,1)$ & $170(45,9)$ & $0,024^{*}$ \\
\hline TENS/Eletroestimulação & $90(29,4)$ & $11(17,2)$ & $179(58,5)$ & $36(56,3)$ & $37(12,1)$ & $17(26,6)$ & $306(82,7)$ & $64(17,3)$ & $0,005^{*}$ \\
\hline Crioterapia & $74(32,9)$ & $27(18,6)$ & $122(54,2)$ & $93(64,1)$ & $29(12,9)$ & $25(17,2)$ & $225(60,8)$ & $145(39,2)$ & $0,005^{*}$ \\
\hline Calor & $62(33,5)$ & $39(21,1)$ & $100(54,1)$ & $115(62,2)$ & $23(12,4)$ & $31(16,8)$ & $185(50)$ & $185(50)$ & $0,024^{*}$ \\
\hline Turbilhão & $66(37,3)$ & $35(18,1)$ & $92(52)$ & $123(63,7)$ & $19(10,7)$ & $35(18,1)$ & $177(47,8)$ & $193(52,2)$ & $0,000^{*}$ \\
\hline Exercícios aeróbios & $56(27,2)$ & $45(27,4)$ & $121(58,7)$ & $94(57,3)$ & $29(14,1)$ & $25(15,2)$ & $206(55,7)$ & $164(44,3)$ & 0,942 \\
\hline Exercícios de solo & $50(24,5)$ & $51(30,7)$ & $126(61,8)$ & $89(53,6)$ & $28(13,7)$ & $26(15,7)$ & $204(55,1)$ & $166(44,9)$ & 0,276 \\
\hline Fortalecimento muscular & $100(27,9)$ & $1(8,3)$ & $209(58,4)$ & $6(50)$ & $49(13,7)$ & $5(41,7)$ & $358(96,8)$ & $12(3,2)$ & $0,018^{*}$ \\
\hline Hidroterapia & $99(27,7)$ & $2(15,4)$ & $206(57,7)$ & $9(69,2)$ & $52(14,6)$ & $2(15,4)$ & $357(96,5)$ & $13(3,5)$ & 0,609 \\
\hline Bengalas e muletas & $47(27,3)$ & $54(27,3)$ & $97(56,4)$ & $118(59,6)$ & $28(16,3)$ & $26(13,1)$ & $172(46,5)$ & $198(53,5)$ & 0,675 \\
\hline Calçados & $69(27)$ & $32(28,1)$ & $155(60,5)$ & $60(52,6)$ & $32(12,5)$ & $22(19,3)$ & $256(69,2)$ & $114(30,8)$ & 0,184 \\
\hline Órteses, braces e palmilhas & $63(27,9)$ & $38(26,4)$ & $131(58)$ & $84(58,3)$ & $32(14,2)$ & $22(15,3)$ & $226(61,1)$ & $144(38,9)$ & 0,928 \\
\hline Ervas medicinais & $26(23,2)$ & $75(29,1)$ & $73(65,2)$ & $142(55)$ & $13(11,6)$ & $41(15,9)$ & $112(30,3)$ & $258(69,7)$ & 0,188 \\
\hline Acupuntura & $70(28,2)$ & $31(25,4)$ & $137(55,2)$ & $78(63,9)$ & $41(16,5)$ & $13(10,7)$ & $248(67)$ & $122(33)$ & 0,198 \\
\hline Kinesio taping & $70(29,8)$ & $31(23)$ & $139(59,1)$ & $76(56,3)$ & $26(11,1)$ & $28(20,7)$ & $235(63,5)$ & $135(36,5)$ & $0,029^{*}$ \\
\hline Educação do paciente & $98(26,8)$ & $3(60)$ & $214(58,6)$ & $1(20)$ & $53(14,5)$ & $1(20)$ & $365(98,6)$ & $5(1,4)$ & 0,198 \\
\hline Repouso & $62(31,5)$ & $39(22,5)$ & $114(57,9)$ & $101(58,4)$ & $21(10,7)$ & $33(19,1)$ & $197(53,2)$ & $173(46,8)$ & $0,028^{*}$ \\
\hline Terapia manual & $83(2,4)$ & $18(26,9)$ & $179(59,1)$ & $36(53,7)$ & $41(13,5)$ & $13(19,4)$ & $303(81,9)$ & $67(18,1)$ & 0,456 \\
\hline
\end{tabular}

ME/E: Muito Eficiente/Eficiente; I/PE: Ineficiente/Pouco eficiente; TENS: Transcutaneous Electrical Nerve Stimulation; Teste qui-quadrado; * $<<0,05 ;$ O número e porcentagem total de participantes é a somatória de respostas ME/E de cada grupo e I/PE de cada grupo, para cada terapia (linha).

A Figura 1 apresenta as razões de chance e os respectivos intervalos de confiança (IC) a 95\% para as terapias que apresentaram diferenças significativas na análise anterior (Tabela 2). Para este momento, considerou-se a categoria mestrado/doutorado como referência para as categorias graduação e especialização/residência. 
Figura 1. Associação entre qualificação profissional e percepção de eficiência ou ineficiência das intervenções com diferenças significativas

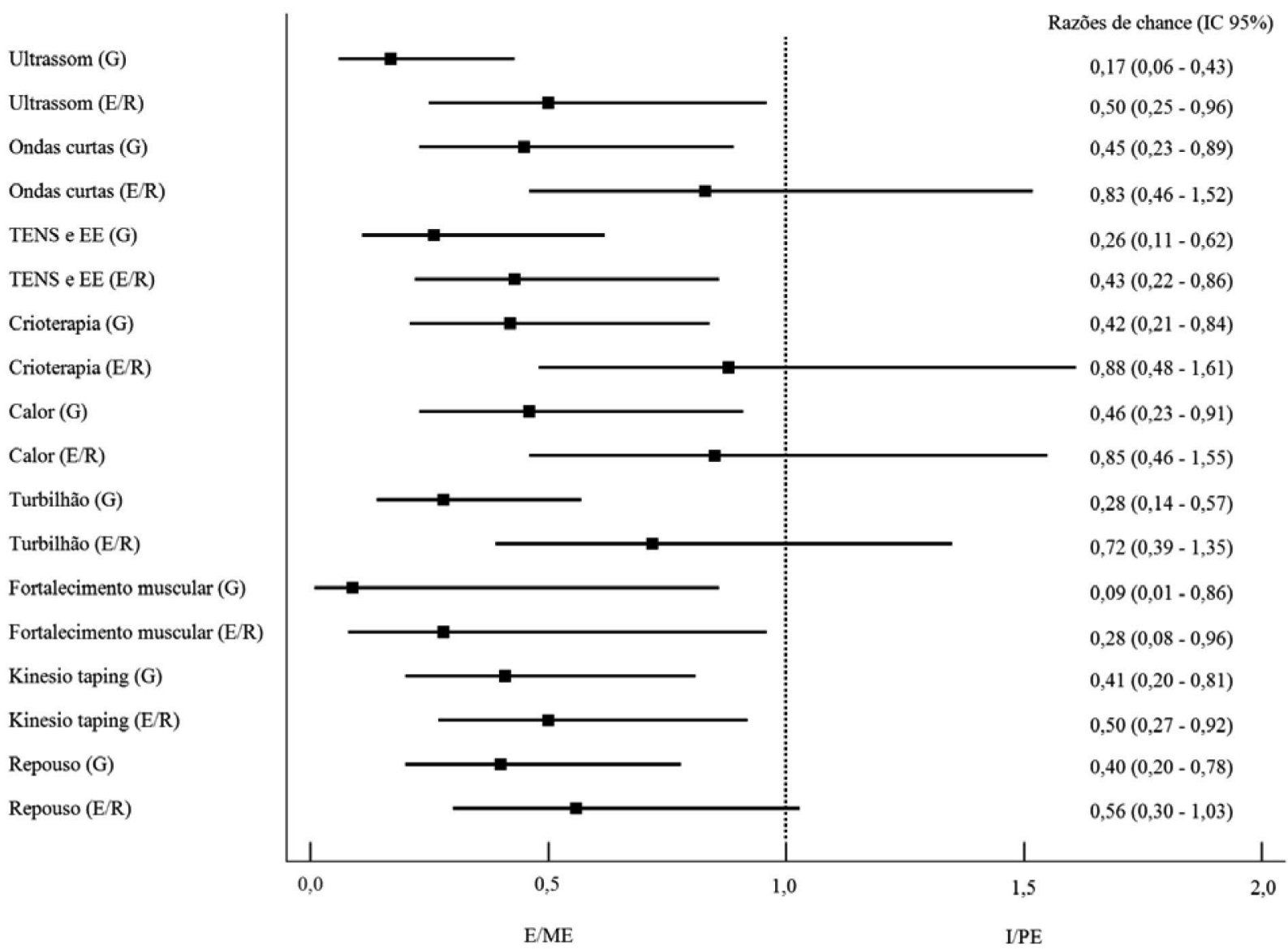

TENS: Transcutaneous electrical nerve stimulation; EE: Eletroestimulação; G: Graduação; E/R:

Especialização/Residência; E/ME: Eficiente/Muito eficiente; I/PE: Ineficiente/Pouco eficiente; Mestrado/Doutorado: categoria de referência.

A análise de regressão logística indicou associação inversa entre a categoria graduação e as intervenções (ultrassom, ondas curtas, TENS e eletroestimulação, crioterapia, calor superficial, turbilhão, fortalecimento muscular, kinesio taping e repouso), sugerindo que possuir tal titulação diminui significativamente as chances em perceber tais terapias como ineficientes ou pouco eficientes para o tratamento da OA de joelho. O mesmo se reproduz para o grupo especialização/residência e as intervenções ultrassom, TENS e eletroestimulação, fortalecimento muscular e o kinesio taping (Figura 1).

Para as outras terapias listadas no inquérito, não houve diferenças significativas. 


\section{Discussão}

Os resultados deste inquérito com 370 fisioterapeutas que atuam na região Norte do Brasil indicam que existe associação entre qualificação profissional e o que estes avaliam como intervenções eficientes ou ineficientes para tratar OA de joelho. Nossos achados mostraram que todas as intervenções referentes a eletroterapia e termoterapia, exceto Laser, estavam associadas com a qualificação profissional do fisioterapeuta, especialmente entre profissionais graduados. Aparentemente, apesar da recente evidência indicando baixa efetividade destas técnicas 12,13 , a crença em terapias passivas com o uso de equipamentos continua a permear a base teórica de muitos profissionais, o que pode sugerir que a Prática Baseada em Evidência (PBE) não é corretamente abordada ou adequadamente implementada em disciplinas da graduação nesta região do Brasil.

A interpretação, por uma parcela de fisioterapeutas, da eletroterapia e ultrassom como modalidades muito utilizadas e eficientes observada em nosso estudo não é um dado inédito. Walsh e Hurley constataram que $66 \%$ de um grupo de fisioterapeutas do Reino Unido citaram as modalidades eletroterapêuticas como a segunda intervenção mais utilizada para tratar OA de joelho ${ }^{\frac{14}{4}}$. Somado à eletroterapia, programas de exercícios terapêuticos também foram mencionados como intervenções frequentes em nosso estudo. Estes, no entanto, são reconhecidos como terapias eficientes para o tratamento de OA por aumentar a força muscular, amplitude de movimento e a capacidade aeróbia, e consequentemente melhorar a dor, função e qualidade de vida dos pacientes ${ }^{15}$. O fortalecimento muscular, exercício largamente recomendado $16-18$, mostrou-se fortemente associado a fisioterapeutas graduados e especialistas. Felizmente, este aspecto indica que a intervenção é reconhecida como muito eficiente ou eficiente por quase todos os respondentes.

Surpreendentemente, kinesio taping e repouso foram consideradas intervenções eficientes por $63,5 \%$ e $53,2 \%$ dos participantes, respectivamente. Diferentemente de outro inquérito com fisioterapeutas do Reino Unido, onde estas intervenções foram as menos utilizadas ${ }^{19}$. Além disso, fisioterapeutas graduados apresentaram $60 \%$ menos chance de considerar repouso uma intervenção pouco eficiente ou ineficiente para o tratamento de OA de joelho. Isto é preocupante, pois indica que parte dos graduados pode estar recomendando e utilizando repouso como tratamento, mesmo que esta conduta seja amplamente conhecida como ineficiente e prejudicial. As recomendações clínicas suportam exatamente o contrário, recomendando programas regulares de exercício físico por auxiliar no controle dos sintomas e peso corporal sem o risco de efeitos colaterais de medicamentos ${ }^{20}$.

A tomada de decisão clínica é um tópico de frequente interesse entre clínicos e pesquisadores. Da Costa et al. investigaram como fisioterapeutas decidiam sobre as intervenções utilizadas e identificaram que profissionais que seguiam os princípios da PBE eram mais propensos a utilizar exercícios terapêuticos e educação e menos propensos a utilizar ultrassom e eletroterapia ${ }^{10}$.

Nós enfatizamos que o tempo médio de atividade profissional em nosso estudo foi em média 6,8 anos e, de acordo com as respostas, estes profissionais não estão atualizados com a melhor evidência sobre tratamento de OA de joelho. É extremamente importante que profissionais de saúde continuem a se atualizar com cursos voltados para áreas específicas, como a musculoesquelética, e na interpretação de diretrizes de prática clínica ${ }^{21,22}$ e artigos científicos, baseando as condutas em Fisioterapia na melhor evidência científica disponível e reduzindo o risco de utilizar tratamentos que não trazem benefícios aos pacientes.

No contexto de treinamento profissional no Brasil, entendemos que, talvez, seja necessário o aprimoramento do ensino nos cursos de graduação com estratégias de ensino com foco na maior participação dos estudantes e professores. A inclusão destas estratégias melhora o aprendizado e encoraja o desenvolvimento profissional23,24. O Brasil é um país com grande dimensão territorial e diversidade cultural e econômica. A distância entre cidades da região Norte e regiões brasileiras mais desenvolvidas economicamente e limitado acesso à internet em algumas municipalidades desta região podem contribuir para as discrepâncias entre a percepção de eficiência de algumas terapias e recomendações de diretrizes de prática clínica para o tratamento da doença encontradas neste estudo. 
Um dos pontos fortes do estudo é que o tratamento fisioterapêutico foi avaliado utilizando um número relevante de possíveis intervenções. Este inquérito destaca resultados que podem ajudar a identificar e compreender o julgamento dos fisioterapeutas desta região do Brasil sobre o uso e eficiência de terapias, possíveis lacunas na formação ou nos métodos de atualização profissional. Pode ainda auxiliar na reavaliação e reformulação das formas e conteúdos desenvolvidos nas disciplinas que formam o aluno acerca das condutas terapêuticas em doenças reumatológicas e musculoesqueléticas. Novas pesquisas são necessárias para tentar identificar a causa destas discrepâncias entre o que o profissional considera eficiente e utiliza na clínica diária e o que as evidências suportam como melhores recomendações terapêuticas e estimular a PBE entre estes profissionais.

A pesquisa apresenta limitações. Trata-se de um estudo transversal, que não permite determinar relações causais para as discrepâncias observadas. A coleta de dados por autorrelato pode contribuir para respostas excessivamente positivas dos profissionais em relação às intervenções para tratar OA de joelho, caracterizando o viés de desejabilidade social. Não podemos afirmar que isso aconteceu, pois nenhum instrumento foi utilizado para avaliar este viés, como sugerido por Perinelli e Gremigni ${ }^{25}$.

\section{Conclusão}

Existe associação entre o nível de qualificação profissional de fisioterapeutas na região Norte do Brasil e sua avaliação de eficiência ou ineficiência de intervenções para OA de joelho. Estes resultados indicam que, quanto menor o nível acadêmico do profissional, menores são as chances de fisioterapeutas perceberem como pouco eficiente, ou mesmo ineficiente, intervenções terapêuticas sabidamente não recomendadas para o tratamento desta doença.

\section{Contribuições dos autores}

Todos os autores contribuíram substancialmente para a construção do projeto, coleta de dados, análise e interpretação de dados, redação do artigo, revisão e aprovação final da versão a ser publicada.

\section{Conflitos de interesses}

Nenhum conflito financeiro, legal ou político envolvendo terceiros (governo, empresas e fundações privadas, etc.) foi declarado para nenhum aspecto do trabalho submetido (incluindo, mas não se limitando a subvenções e financiamentos, participação em conselho consultivo, desenho de estudo, preparação de manuscrito, análise estatística, etc.).

\section{Referências}

1. Glyn-Jones S, Palmer AJR, Agricola R, Price AJ, Vincent TL, Weinans H, et al. Osteoarthritis. Lancet. 2015;386(9991):376-87. https://doi.org/10.1016/S0140-6736(19)30417-9

2. Plotnikoff R, Karunamuni N, Lytvyak E, Penfold C, Schopflocher D, Imayama I, et al. Osteoarthritis prevalence and modifiable factors: A population study Chronic Disease epidemiology. BMC Public Health. 2015;15(1):1195. https://dx.doi. org/10.1186\%2Fs12889-015-2529-0

3. Busija L, Bridgett L, Williams SRM, Osborne RH, Buchbinder $\mathrm{R}$, March L, et al. Osteoarthritis. Best Pract Res Clin Rheumatol. 2010;24(6):757-68. https://doi.org/10.1016/j.berh.2010.11.001

4. Bijlsma JW, Berenbaum F, Lafeber FPJG. Osteoarthritis: an update with relevance for clinical practice. Lancet. 2011;377(9783):2115-26. https://doi.org/10.1016/s01406736(11)60243-2

5. Prieto-Alhambra D, Judge A, Javaid MK, Cooper C, DiezPerez A, Arden NK. Incidence and risk factors for clinically diagnosed knee, hip and hand osteoarthritis: Influences of age, gender and osteoarthritis affecting other joints. Ann Rheum Dis. 2014;73(9):1659-64. https://doi.org/10.1136/ annrheumdis-2013-203355

6. Fransen M, McConnell S, Harmer AR, Esch MVD, Simic M, Bennell KL. Exercise for osteoarthritis of the knee: A Cochrane systematic review. Br J Sports Med. 2015;49(24):1554-7. https:// doi.org/10.1136/bjsports-2015-095424 
7. Kolasinski SL, Neogi T, Hochberg MC, Oatis C, Guyatt G, Block J, et al. 2019 American College of Rheumatology/Arthritis Foundation Guideline for the Management of Osteoarthritis of the Hand, Hip, and Knee. Arthritis Care Res. 2020;72(2):149-62. https://doi.org/10.1002/acr.24131

8. McAlindon TE, Bannuru RR, Sullivan MC, Arden NK, Berenbaum $\mathrm{F}$, Bierma-Zeinstra SM, et al. OARSI guidelines for the nonsurgical management of knee osteoarthritis. Osteoarthr Cartil. 2014;22(3):363-88. https://doi.org/10.1016/j.joca.2014.01.003

9. Holden MA, Nicholls EE, Young J, Hay EM, Foster NE. UK-based physical therapists' attitudes and beliefs regarding exercise and knee osteoarthritis: Findings from a mixed-methods study. Arthritis Rheum. 2009;61(11):1511-21. https://doi.org/10.1002/ $\underline{\text { art.24829 }}$

10. Costa BR, Vieira ER, Gadotti IC, Colosi C, Rylak J, Wylie T, et al. How do physical therapists treat people with knee osteoarthritis, and what drives their clinical decisions? A population-based crosssectional survey. Physiother Can. 2017;69(1):30-7. https://dx.doi. org/10.3138\%2Fptc.2015-83

11. Spitaels D, Hermens R, Van Assche D, Verschueren S, Luyten F, Vankrunkelsven P. Are physiotherapists adhering to quality indicators for the management of knee osteoarthritis? An observational study. Musculoskelet Sci Pract. 2017;27:112-23. https://doi.org/10.1016/j.math.2016.10.010

12. Geenen $\mathrm{R}$, Overman $\mathrm{CL}$, Christensen $\mathrm{R}$, Åsenlöf $\mathrm{P}$, Capela $\mathrm{S}$, Huisinga $\mathrm{KL}$, et al. EULAR recommendations for the health professional's approach to pain management in inflammatory arthritis and osteoarthritis. Ann Rheum Dis. 2018;77(6):797-807. https://doi.org/10.1136/annrheumdis-2017-212662

13. Zeng C, Li H, Yang T, Deng ZH, Yang Y, Zhang Y, et al. Electrical stimulation for pain relief in knee osteoarthritis: Systematic review and network meta-analysis. Osteoarthr Cartil. 2015;23(2):189-202. https://doi.org/10.1016/j.joca.2014.11.014

14. Walsh NE, Hurley MV. Evidence based guidelines and current practice for physiotherapy management of knee osteoarthritis. Musculoskeletal Care. 2009;7(1):45-56. https://doi.org/10.1002/ msc.144

15. Yamato TP, Deveza LA, Maher CG. Exercise for osteoarthritis of the knee (PEDro synthesis). Br J Sports Med. 2016;50(16):1013-4. http://dx.doi.org/10.1136/bjsports-2016-096104

16. Bartholdy C, Juhl C, Christensen R, Lund H, Zhang W, Henriksen $M$. The role of muscle strengthening in exercise therapy for knee osteoarthritis: A systematic review and metaregression analysis of randomized trials. Semin Arthritis Rheum. 2017;47(1):9-21. https://doi.org/10.1016/j.semarthrit.2017.03.007
17. Brosseau L, Taki J, Desjardins B, Thevenot O, Fransen M, Wells GA, et al. The Ottawa panel clinical practice guidelines for the management of knee osteoarthritis. Part two: Strengthening exercise programs. Clin Rehabil. 2017;31(5):596-611. https://doi. org/10.1177/0269215517691084

18. Imoto AM, Pardo JP, Brosseau L, Taki J, Desjardins B, Thevenot $O$, et al. Evidence synthesis of types and intensity of therapeutic land-based exercises to reduce pain in individuals with knee osteoarthritis. Rheumatol Int. 2019;39(7):1159-79. https://doi. org/10.1007/s00296-019-04289-6

19. Holden MA, Nicholls EE, Hay EM, Foster NE. Physical Therapists' Use of Therapeutic Exercise for Patients With Clinical Knee Osteoarthritis in the United Kingdom: In Line With Current Recommendations? Phys Ther. 2008;88(10):1109-21. https://doi. org/10.2522/ptj.20080077

20. Bannuru RR, Schmid CH, Kent DM, Vaysbrot EE, Wong JB, McAlindon TE. Comparative Effectiveness of Pharmacologic Interventions for Knee Osteoarthritis: a systematic review and network meta-analysis. Ann Intern Med. 2015;162(1):46-54. https://doi.org/10.7326/m14-1231

21. Davis D. Continuing education, guideline implementation, and the emerging transdisciplinary field of knowledge translation. J Contin Educ Health Prof. 2006;26(1):5-12. https://doi.org/10.1002/ chp. 46

22. Li LC, Hurkmans EJ, Sayre EC, Vliet Vlieland TPM. Continuing Professional Development Is Associated With Increasing Physical Therapists' Roles in Arthritis Management in Canada and the Netherlands. Phys Ther. 2010;90(4):629-42. https://doi. org/10.2522/ptj.20080409

23. Marques LMNSR. Active methodologies as strategies to develop education in values in nursing graduation. Esc Anna Nery. 2018;22(3):1-6. https://doi.org/10.1590/2177-9465-ean-2018-0023

24. Simon E, Jezine E, Vasconcelos EM, Ribeiro KSQS. Active teaching-learning methodologies and popular education: agreements and disagreements in the context of health professionals' educationos. Interface Commun Heal Educ. 2014;18(Supl.2):1355-64. https://doi.org/10.1590/1807$\underline{57622013.0477}$

25. Perinelli E, Gremigni P. Use of Social Desirability Scales in Clinical Psychology: A Systematic Review. J Clin Psychol. 2016;72(6):534-51. https://doi.org/10.1002/jclp.22284 